\title{
CODE SWITCHING IM FACHSPRACHLICHEN UNTERRICHT DER NOTFALLMEDIZINISCHEN KOMMUNIKATION
}

\author{
GRZEGORZ LISEK \\ Universität Greifswald \\ grzegorz.lisek@uni-greifswald.de \\ ORCID: 0000-0003-1613-3855
}

\section{ZUSAMMENFASSUNG}

Code Switching ist kein neues Phänomen. Interessant bleibt nach wie vor jedoch, welche Faktoren oder Situationen haben überwiegende Bedeutung beim Eintreten dieser Erscheinung. Ein notfallmedizinisches Simulationstraining, welches hier den Rahmen der Untersuchung gibt, ist eine gute Möglichkeit um medizinische Kommunikation in den Fokus zu nehmen. Notfallmedizinische Kommunikation in einer Nachbarsprache bedarf besonderer Überlegung. Im Rahmen des Projekts InGRiP wird die notfallmedizinische Kommunikation deutschsprachiger Rettungskräfte beobachtet und ausgewertet. Da Polnisch nicht als die erste Fremdsprache gelernt wird, wird auch Code Switching vermutet. Die Beobachtungen haben gezeigt, dass die Rettungskräfte, die Russisch bereits gelernt haben, Code Switching insbesondere bei den Zahlen betreiben. Es kommt auch zum Sprachwechsel ins Englische oder in die Erstsprache Deutsch, jedoch viel seltener als im Falle von Russisch. Das hier beobachtete Code Switching ins Russische ist ein nicht-funktionales Code Switching, das von den Rettungskräften unbewusst betreiben wird. Die Ursache wird in dem Sprachproduktionsprozess vermutet.

SCHLÜSSELWÖRTER: Notfallkommunikation, Nachbarsprache, Code Switching, Polnisch, Russisch.

\section{CODE SWITCHING IN THE LANGUAGE TEACHING OF EMERGENCY MEDICAL COMMUNICATION}

\section{ABSTRACT}

Code switching is not a new phenomenon. However, it is still interesting to know which factors or situations are predominant in the occurrence of this phenomenon. Emergency medical simulation training, which provides the framework for this study, is a good way to focus on medical communication. Emergency medical communication in a neighbour's language requires special consideration. In the InGRiP project, the emergency medical communication of Germanspeaking paramedics is being observed and evaluated. Since Polish is not learned as the first foreign language, code switching is also suspected. Observations have shown that the rescue workers who have already learned Russian engage in code switching, especially when it comes to numbers. They also switch to English or German, their first language, but much less frequently than in the case of Russian. The code switching into Russian observed here is a non-functional code switching that the rescuers perform unconsciously. The cause is assumed to be the language production process.

KEYWORDS: emergency communication, neighbour's language, code switching, Polish, Russian. 
Wie wichtig, die Kenntnisse einer Fremdsprache sind, entdecken wir jedes Mal, wenn wir in ein Land verreisen, dessen Sprache wir nicht sprechen. Bei einem Notfall sind zwar medizinische Kenntnisse wichtig, die Fremdsprache - genauer gesagt die medizinische Kommunikation in der Fremdsprache - kann jedoch insbesondere in Grenzregionen eine große Rolle spielen. Dies gilt vor allem dann, wenn die Rettungsteams international zusammengesetzt sind oder gar aus dem Nachbarland ausrücken. Der zweite Fall trifft auf das internationale notfallmedizinische Projekt InGRiP ${ }^{1} \mathrm{zu}$, dass bis Februar 2021 von dem Institut für Slawistik wissenschaftlich begleitet wurde.

Im Zentrum des vorliegenden Beitrags steht Code Switching, hier als Phänomen fremdsprachiger Notfallkommunikation kontextualisiert. Am Beispiel der Beobachtungen aus den Simulationstrainings im Rahmen des polnischen Tertiärsprachenunterrichts für deutschsprachige Rettungskräfte soll die folgende Forschungsfrage beantwortet werden: Inwieweit wird die notfallmedizinische Kommunikation in der Drittsprache (hier: Polnisch) während eines Simulationstrainings durch die Erstsprache oder bereits gelernte Fremdsprachen beeinflusst? Um die Frage beantworten zu können, werden das Vorhandensein und das Ausmaß von Code Switching deutschsprachiger Rettungskräfte beobachtet, erfasst und charakterisiert. Die Untersuchung ist von der Annahme geleitet, dass Teilnehmende in einer Stresssituation, die ein Simulationstraining hervorruft, auf bereits vorhandene, besser im mentalen Lexion abgespeicherte (Fremd)sprachkenntnisse zurückgreifen, und zwar insbesondere auf eine der Tertiärsprache nahen Sprache (hier: Russisch²).

Der Beitrag besteht aus drei Abschnitten. Die Haupttermini der Untersuchung werden im ersten Abschnitt festgehalten. Im zweiten Abschnitt werden das Datenmaterial sowie das Forschungsdesign erläutert. Im Anschluss daran, also im dritten Abschnitt, werden die Ergebnisse der Beobachtungen zusammengefasst und im Hinblick auf die Annahmen diskutiert. Den Rahmen der vorliegenden Analyse schafft der Simulationstraining, der als Abschluss des fachsprachlichen Kurses der notfallmedizinischen Kommunikation in der Nachbarsprache Polnisch und eine Art der Überprüfung des Kursprogramms gilt.

1 Nähere Informationen zum Projekt Integrierter grenzüberschreitender Rettungsdienst Pomerania/Brandenburg (InGRiP), das in den Jahren 2017-2021 realisiert wurde, sowie zu den beteiligten Partnern finden Sie unter https://www.medizin.uni-greifswald.de/ingrip/de/home/ (letzter Zugriff: 15.10.2021).

${ }^{2}$ Vor dem Beginn der Sprachkurse wurden mögliche Sprachkenntnisse ins Auge gefasst. Die Ergebnisse der Analyse belegen, dass das Russische für Rettungssanitäter auf beiden Seiten der Grenze eine bekannte Sprache ist: 56 \% der polnischen Rettungssanitäter und $51 \%$ der deutschen Rettungssanitäter hatten in der Schule Russisch als Fremdsprache. 


\section{NOTFALLMEDIZINISCHE KOMMUNIKATION WÄHREND DES SIMULATIONSTRAININGS}

Simulationen oder Simulationstrainings sind in der Sprachdidaktik nichts Unübliches. Bereits Studierende werden in simulationsähnlichen Settings unterrichtet (vgl. Klčová 2019), da „Fallstudien, Szenarien, Planspiele und Simulationen zu den interaktiven Lehr- und Lernmethoden [gehören]“ (Illés und Kegyes 2019: 120). Dies soll den Erfolg eines Sprachkurses erhöhen und auf die Kompetenzentwicklung der Teilnehmenden unterstützend wirken. Darüber hinaus sind Simulationen „Lernprojekte, die nicht nur Lernerfolge garantieren, sondern auch zu einer experimentierenden Lösung eines fachlichen Problems motivieren“ (Illés und Kegyes 2019: 121). Vor allem um Lernerfolge ging es beim Simulationstraining der deutschen Rettungskräfte. Sie sollen notfallmedizinische Kommunikation mit polnischsprachigen Patienten üben und ihre Sprachfertigkeiten überprüfen. Rettungskräfte können somit praktisch erleben, dass eine Fremdsprache zu lernen, viel mehr als nur Vokabeln und Wendungen $\mathrm{zu}$ lernen bedeutet. Während einer Simulation wird den Teilnehmenden bewusst, dass „Lernen erheblich mehr als die Ansammlung von Wissen [ist]“ (Riedl 2012: 114).

Im Vordergrund des Simulationstrainings steht die (notfall)medizinische Kommunikation, die von kommunikativen Handlungen ${ }^{3}$ und Rollen geprägt ist. Die Kommunikation kann somit , als funktionale Gesprächssituation bezeichnet [werden], d.h. das Gespräch zwischen den Akteuren hat eine konkrete Funktion - hier eine adäquate medizinische Patientenversorgung."

In jeder medizinischen Kommunikation agieren Rettungssanitäter und Notärzte sowie die Patienten als Akteure des Gesprächs. Beim Code Switching ist davon auszugehen, dass verschiedene soziale Rollen der Sprechenden aktiviert werden können (vgl. Riehl 2014: 103). Unabhängig von den Rollen: Für das Gelingen der Kommunikation ist der Erwerb grundlegendär kommunikativer Äußerungsmuster auf Polnisch ${ }^{4}$ essentiell. $\mathrm{Zu}$ den Äußerungsmustern gehören Fragen, ${ }^{5}$ Bitten oder Aufforderungen.

${ }^{3}$ Unter der Formulierung kommunikatives Handeln werden in diesem Beitrag vor allem Sprechakte (vgl. Searle 1997: 39) verstanden. Da im notfallmedizinischen Fremdsprachenunterricht Sprechhandlungen realisiert, die beispielsweise Beschwerden oder ihre Intensität thematisieren, bedeutend sind direktive, erotetische, assertive und expressive Sprechakte (vgl. Lisek 2020).

${ }^{4}$ Zum detaillierten Aufbau des Sprachkurses siehe Lisek (vgl. 2019: 23).

5 Diese wurden für die allgemeinmedizinische Kommunikation von Bechmann (vgl. 2014: 190ff.) bereits gesammelt und kategorisiert. In Bezug auf seinen Fragenkatalog sollen hier einzelne notfallmedizinische Kategorien mit Beispielen illustriert werden. Eine wesentliche Rolle spielen daher Informationsfragen (Was ist passiert?) sowie Einschätzungsfragen (Von 0 bis 10, wie stark ist der Schmerz?). 
Das Hauptmerkmal notfallmedizinischer Kommunikation stellen Genauigkeit und Sachlichkeit dar. Die medizinischen Schemata, ${ }^{6}$ die während der Notfallanamnese angewandt werden, tragen $\mathrm{zu}$ Genauigkeit und Sachlichkeit (vgl. Stemmler und Hecker 2017: 100) bei. Die Schemata können im Sinne des Chunkings ${ }^{7}$ bei dem Erwerb der entsprechenden Fertigkeiten in der Fremdsprache unterstützend sein.

\section{NACHBARSPRACHE UND CODE SWITCHING}

Das Erlernen der Nachbarsprache, d.h. einer Sprache mit einem besonderen Stellenwert in der Grenzregion (vgl. Hryniewicz und Lisek 2019), erfolgt im Projekt im Tertiärsprachenunterricht. Dabei ist einzuordnen, dass neben der erworbenen Erstsprache (L1) erfolgt —üblicherweise in der Schulzeit - der Erwerb weiterer Sprachen („Fremdsprachen“). Die Erwerbsmechanismen beim Erst- und Fremdspracherwerb unterscheiden sich voneinander (vgl. Meisel 2011:3). Wiederum wird die zuerst gelernte Fremdsprache als Zweitsprache (L2) bezeichnet. Eine der später gelernten Fremdsprachen wird folglich als Tertiärsprache (L3) bezeichnet (vgl. Hammarberg 2010: 97). Beim Tertiärspracherwerb ergeben sich durch Fremdsprachenlernererfahrungen und -strategien (vgl. Hufeisen und Lindemann 1998: 171) weitere qualitative Unterschiede zum L2-Erwerb. In unserem Falle ist Polnisch zwar soziolinguistisch gesehen eine Nachbarsprache, erwerbshistorisch vor allem aber eine Tertiärsprache, da die Kursteilnehmenden bereits entweder Englisch oder/und Russisch gelernt haben.

Als Tertiärsprachenunterricht wird also in diesem Artikel ein (gesteuerter) Lernprozess gemeint, während dessen keine erste Fremdsprache unterrichtet wird. Darüber hinaus ergeben sich im Unterricht mögliche vielfältige Rückgriffe auf sprachliches Wissen aus dem Russischen, da Russisch und Polnisch eng verwandte Sprachen sind.

Diese Rückgriffe können sich in Code Switching ${ }^{8}$ zeigen. Das Phänomen wird hier aus einer soziolinguistischen Perspektive betrachtet. Blom und

\footnotetext{
${ }^{6}$ Schemata der Notfallanamnese, die als Akronyme den Rettungskräften bekannt sind, stellen Fragehandlungen dar. Mögliche Fragen des OPQRST-Schemas lauten wie folgt: $(\mathrm{O})$ Wann haben die Schmerzen begonnen? (P) Wann tut es mehr und wann weniger weh? (Q) Was ist das für ein Schmerz? (R) Strahlt der Schmerz aus? (S) Von 0 bis 10, wie stark sind die Schmerzen? (T) Sind die Schmerzen jetzt stärker?

7 Chunking ist nach Roche (vgl. 2013: 116) die automatisierte Wiedergabe von "Mehrworteinheiten, Formeln oder Redewendungen", hier verstanden als fertige FrageAntwort-Sequenzen.

8 An dieser Stelle wird aus Platzgründen auf eine detaillierte Auseinandersetzung mit Begrifflichkeiten wie Interferenz, Transfer und Code Switching verzichtet. Für diesen Beitrag gilt: Interferenz die allgemeinste Bezeichnung für Erscheinungen des Fremdsprachenerwerbs bei Lernenden. Transfer ist als eine der Erscheinungen zu verstehen, die sich durch ein festes
} 
Gumperz (vgl. 1972: 424) sowie Gumperz (vgl. 1982: 92) zufolge kann zwischen situativem und metaphorischem Code Switching differenziert werden. Im Falle eines situativen Code Switchings besteht eine direkte Verbindung zwischen einer bestimmten Sprache oder Varietät und einer konkreten Sprachsituation. Zum situativen Sprachwechsel kommt es demzufolge aufgrund von äußeren Faktoren, wie beispielsweise Wechsel der Gesprächspartner oder Situationsteilnehmer. Zum metaphorischen Code Switching kommt es hingegen, wenn der Sprachwechsel bspw. durch Themenwechsel oder subjektive Umstände und Motivationen von Sprecherinnen und Sprechern verursacht wird. Die Unterscheidung zwischen situativem und metaphorischem Typ kann wie folgt charakterisiert werden: Der situative Typ liegt dann vor, wenn die Sprachenwahl mit dem sozialen Rahmen in Verbindung steht. So kann der Sprachwechsel z. B. den Übergang von geschäftlichen zu privaten Themen während eines Gesprächs markieren oder ein Codewechsel von einer substandardsprachlichen zur standardsprachlichen Varietät erfolgen, wenn räumliche Grenzen passiert werden. Der metaphorische Typ des Code Switchings dient dazu, zusätzliche Informationen zu kommunizieren, z. B. um der Äußerung einen besonderen Stil oder einen anekdotischen Charakter zu verleihen.

Im Falle notfallmedizinischer Kommunikation beim Simulationstraining wird angenommen, dass die Rettungskräfte vor allem auf situatives Code Switching zurückgreifen, da es um die Kommunikation essentieller Inhalte geht. Des Weiteren kann zwischen adressaten- oder diskursabhängigem Code Switching unterschieden werden (vgl. Auer 1988). ${ }^{9}$

Aus der fremdsprachendidaktischen Perspektive ist zu unterstreichen, dass Code Switching beim Erlernen von sowohl nah verwandten (Polnisch - Russisch) als auch weniger verwandten (Polnisch - Englisch) Sprachen vorkommen kann. Beim Erwerb einer Tertiärsprache kann es zu weiteren Code SwitchingKonstellationen kommen. In der Fachliteratur (vgl. Auer 1988: 196) wird der Auslöser für Code Switching in der Bevorzugung einer Sprache durch den Sprecher oder Adressaten gesehen. Dieser Grund ist in der vorliegenden Fallstudie ebenso wichtig, da wir uns hier auf dem Niveau der elementaren Sprachverwendung (A1) in der Nachbarsprache Polnisch bewegen. Der Prozess

Sprachprodukt manifestiert. Dagegen Code Switching hat einen gelegentlichen Charakter und kann sicherlich nicht mit Transfer gleichgesetzt werden.

${ }^{9}$ Die definitorische Differenzierung der Begriffe könnte wie folgt aussehen: Wenn eine deutschsprachige Rettungskraft gerade noch mit ihrem deutschen Kollegen gesprochen hat, sich jetzt aber dem polnischen Patienten zuwendet, der kein Deutsch versteht, und auf Englisch den Satz anfängt, dann findet ein adressatenspezifischer Sprachwechsel statt. Nach Auer (1988: 196) als participant-related switching bezeichnet. Zugleich findet ein sprecherzentrierter Sprachwechsel, speaker-related switching, statt d.h. der Sprecher kann sich weniger gut auf Polnisch ausdrücken, weil er nur basale Kompetenzen hat. Wenn wiederum die Rettungskraft durch Benutzung des Englischen eine neue Sprechphase markieren oder das Thema wechseln möchte, handelt es sich nach Auer (1988: 199) um discourse-related switching. 
der Sprachproduktion, auf den als Quelle des Code Switchings Riehl (vgl. 2014: 103) hinweist, ist hier ebenfalls zu beachten.

Somit kann eine weitere Differenzierung des Code Switchings vorgenommen werden, nämlich in funktionales und nicht-funktionales. Beim funktionalen Code Switching, ähnlich wie bei dem situativen Code Switching, kommt es zum Wechsel der Sprache aufgrund von äußeren Faktoren. Beim nichtfunktionalen Code Switching spielt der interner Prozess der Sprachproduktion eine wesentliche Rolle. Deswegen kann auch angenommen werden, dass es sich an einigen Stellen der notfallmedizinischen Kommunikation —bei Zahlen oder Bezeichnung für Organe und Körperteile- um zu der obigen Kategorie gehörendes nicht-funktionales Code Switching handelt.

\section{FORSCHUNGSDESIGN UND DATENMATERIAL}

Dieser Aufsatz basiert auf sprachlichem Handeln, das während des Simulationstrainings im März 2020 im deutsch-polnischen Simulationszentrum in Świnoujście beobachtet wurde. Bereits während der im Unterricht eingesetzten simulationsähnlichen Rollenspiele konnte Code Switching ins Russische ausgemacht werden (vgl. Lisek 2021).

Im Vordergrund dieser Untersuchung steht die Rolle von bereits gelernten (nicht) slawischen Sprachen für die notfallmedizinische Kommunikation im Allgemeine und insbesondere während der Simulationstrainings: Nutzen die Teilnehmenden vorhandene Fremdsprachenkenntnisse und resultiert dies auch in Code Switching? Wenn es zu Code Switching kommt, hat dies Auswirkungen auf die notfallmedizinische Kommunikation?

Für die Beobachtung der notfallmedizinischen Kommunikation und der Rolle von früher erworbenen Sprachen bei dem Simulationstraining ergeben sich zwei weitere Fragen:

a) In welche Sprachen wird jeweils gewechselt?

b) Wird bevorzugt in eine Sprache geswitcht?

Um auf die Fragen einzugehen, wurde das sprachliche Handeln ${ }^{10} \mathrm{im}$ Rahmen des internationalen Simulationstrainings für deutsche und polnische Rettungskräfte in Świnoujście (Polen) beobachtet. Es wurden verschiedene notfallmedizinische Szenarien mit einer ca. 5-minütigen Vorbereitungszeit für die Teilnehmenden simuliert. Die allgemeine Simulationsaufgabe bestand darin, eine Notfallanamnese zu Symptomen, Allergien und Vorerkrankungen durchzuführen. Des Weiteren, wenn die Betroffenen ansprechbar waren, sollten Informationen zum Beginn, der Stärke sowie dem Charakter des Schmerzes erfasst werden. Um die Vergleichbarkeit zu gewährleisten, betrafen die einzelnen Aufgaben sich wiederholende medizinische Themen (Atemnot, Beschwerden des Herzkreislaufsystems, anaphylaktischen Schock etc.). Auch die

\footnotetext{
${ }^{10}$ In diesem Artikel wird nur das sprachliche Handeln der deutschen Rettungskräfte fokussiert.
} 
sprachliche Realisierung der standardisierten Übergabe von Patienten ${ }^{11}$ an polnische Kollegen wurde während der Simulation getestet. Da in der notfallmedizinischen Kommunikation etablierte Notfallschemata angewandt werden, ergibt sich eine gewisse Vorhersehbarkeit und Vergleichbarkeit der sprachlichen Daten.

Im Rahmen des Forschungsprojekts „InGRiP“ während des Simulationstrainings wurden interne, wissenschaftliche, nicht-teilnehmende, nicht-standardisierte, direkte Beobachtungen als Laborbeobachtung mit Stimulus, mittels manueller Datenerhebung durchgeführt. $\mathrm{Zu}$ bedenken ist dabei, dass ,jede Beobachtung selektiv und fokussierend [ist], da niemand ein Geschehen vollständig erfassen kann" (Gehrau 2017: 85). Andererseits wird die Beobachtung zwar fokussiert durchgeführt, aber es ist ebenso wichtig auf die mögliche "Betriebsblindheit" des Forscherteams und insbesondere des Beobachtenden $\mathrm{zu}$ achten. Als mögliche Merkmale, die beobachtet werden können, nennt die Fachliteratur (vgl. Gehrau 2017: 84) Folgendes:

a) verbale Seite des Handelns;

b) paraverbale und nonverbale Aspekte (mit dem Körper verbundene Mimik, Gestik und Proxemik);

c) Interaktionen im Rahmen einer hierarchischen Struktur

d) sowie sprachliches Handeln unter zeitlichen und räumlichen Aspekten.

Zwar erscheint nicht nur das verbale Handeln, sondern auch Untermauern des Gesagten mit Gesten oder Mimik (non-verbale Kommunikation) oder das gänzliche Wegbleiben des Verbalen und Realisierung der Kommunikation nur nonverbal interessant, aber aus forschungspraktischen Gründen wird die verbale Seite der Kommunikation fokussiert. Dabei handelt es sich konkret um sprachliches Handeln der Ärzte und Rettungssanitäter/-assistenten untereinander sowie mit einem wachen Patienten ${ }^{12}$ oder Zeugen bzw. den Angehörigen.

Bei dem sprachlichen Handeln ist aus der linguistischen Perspektive wichtig auf die lexikalische und die grammatische Ebene einzugehen. Ebenso produktiv erscheint die phonetische Seite der Sprachhandlung zu reflektieren.

Bei der Untersuchung handelt es sich hier um eine Beobachtung mit notierten Teilsequenzen, nicht um ein vollständiges Transkript im sprachwissenschaftlichen Sinne, das einer vollumfänglichen Analyse unterzogen werden konnte. Das sprachliche Handeln wird in Anlehnung an die HIATKonvention notiert. Ausgewählte Teilsequenzen werden in diesem Beitrag präsentiert und analysiert. Um eine Vergleichbarkeit zu ermöglichen, wird eine Simulationssituation fokussiert, d.h. eine Simulation $\mathrm{zu}$ anaphylaktischem

11 Dazu gehören beispielsweise die Angabe des Namens und Alters der Behandelten, Unfallmerkmale, Symptome, Vitalitätsparameter und verabreichte Medikamente.

12 Aus Gründen der besseren Lesbarkeit wird im Text verallgemeinernd das generische Maskulinum verwendet. Diese Formulierungen umfassen gleichermaßen weibliche, männliche sowie diverse Personen; alle sind damit gleichberechtigt angesprochen. 
Schock. Die Beobachtung wird als eine Chance gesehen, die (interkulturelle) Fachkommunikation zu beschreiben und mögliche Fehlerquellen auszumachen, ohne eine feinkörnige gesprächsanalytische Studie durchzuführen.

\section{ERGEBNISSE}

Im Rahmen der Beobachtung konnte Kommunikation deutscher Rettungskräfte, die mit einem ausländischen Patienten Polnisch gesprochen haben, erfasst werden. Die Beobachtungen betrafen zwei Simulationstrainings, die am 4.03. und 11.03.2020 in Świnoujście stattfanden.

\section{a) Simulation Nr. 1}

Die deutschen Rettungskräfte trafen bei einem Patienten ein, der von einer Wespe gestochen wurde. Der Patient klagte über massive Atemnot und geschwollene Zunge. Die Rettungskräfte versuchten erstmal den Patienten auf Deutsch zu begrüßen [Hallo!]. Sobald sie realisieren, dass es sich um einen polnischsprachigen Patienten handelt, beginnen sie mit dem Anamneseschema auf Polnisch [Co panu teraz dolega?], das mehrheitlich unproblematisch realisiert wird. Es wurden insgesamt 29 Äußerungen erfasst, die im Gespräch mit dem Patienten sowie dem Notarzt durch die Rettungskräfte produziert wurden. Parallel dazu folgten medizinische Tätigkeiten.

Es sind zwei Rettungskräfte anwesend gewesen, eine Rettungskraft ist bilingual (deutsch-polnisch) aufgewachsen und in den abgebildeten Sprechsequenzen als Rettungskraft 2 vermerkt. Der Redeanteil dieser Rettungskraft ist vor allem bei der Anamnese sichtbar und aus der fremdsprachendidaktischen Perspektive weniger interessant, da eine ausgeprägte Sprachkompetenz vorausgesetzt wird. Deswegen liegen auch keine notierten Sequenzen vor. Die Rettungskraft, die tatsächlich an der Sprachschulung teilgenommen und als alleinige Erstsprache Deutsch hat, wurde in den abgebildeten Sprechsequenzen als Rettungskraft ${ }_{1}$ vermerkt.

Allgemein ist $\mathrm{zu}$ bemerken, dass die Teilenehmenden, darunter der Erstsprecher des Deutschen sehr bemüht waren, die Nachbarsprache Polnisch zu benutzen. Dies gelang denen dann insbesondere gut, als sie grammatisch vollständige und inhaltlich korrekte Sätze bildeten. Auch bei elliptischen Äußerungen konnte die Kommunikation trotz vieler Unregelmäßigkeiten in Form von Weglassen von Satzgliedern oder des Codes Switchings erfolgreich und zum Wohl des Patienten aufrechterhalten werden.

Charakteristisch für die beobachtete notfallmedizinische Kommunikation war ein häufiges Code-Switching. Dies wurde erfasst sowohl im Gespräch mit dem Patienten als auch mit dem Notarzt. Die eine Rettungskraft hat insbesondere oft ins Russische gewechselt, dann folgten das Englische und das Deutsche. 


\begin{tabular}{|l|l|}
\hline Rettungskraft1: & My pamagiem, [korr.] my pamożem [rus.] [Wir helfen.] \\
\hline Rettungskraftı: & My jechamy do spitale (sic!). [Wir fahren ins Krankenhaus] \\
\hline Patient: & Świnoujście? \\
\hline Rettungskraftı: & Da [rus.], to [eng.] Swinoujscie [Ja, nach Swinemünde] \\
\hline Rettungskraftı: & Gdzie wy damoj? (sic!) [rus.] [Wo wohnen Sie?] \\
\hline Patient: & [Beklagt schmerzen] \\
\hline Rettungskraft1: & My pamożem [rus.] [Wir helfen.] \\
\hline Rettungskraft1: & Proszę [Hier bitte] \\
\hline Rettungskraftı: & Alles gut [deut.] \\
\hline Patient: & [spricht undeutlich und leise] \\
\hline Rettungskraft1: & Jeszjo raz, Jeszjo raz! [rus.] [Noch einmal] \\
\hline Rettungskraft2: & Proszę raz jeszcze. [Bitte wiederholen Sie noch einmal] \\
\hline
\end{tabular}

TABELLE 1. Gespräch mit Patienten vom 4.03.2020

Die hier abgebildete notfallmedizinische Kommunikation zeigt, wie die gelernten Fremdsprachen oder die Erstsprache in einem Simulationstraining zur Geltung kommen kann. Insbesondere präsent ist das Russische, was zwar auch nicht fehlerfrei ist —was die Markierung (sic!) anzeigt, jedoch an Stelle des Polnischen tritt. Englisch und Deutsch kommen auch stellenweise auch vor.

Es ist davon auszugehen, dass in einer solchen starken Stresssituation, die die Simulation nicht nur imitiert, sondern auch hervorruft, kommt es zu Störungen in der Sprachproduktion und beim Zurückgreifen auf das mentale Lexikon, wo das Polnische anscheinend noch nicht so fest wie das Russische verankert ist. Aus Gesprächen mit der Rettungskraft ist bekannt, dass er über mehrere Jahre Russisch gelernt hat. Dies steht in keinem Vergleich zu den wenigen Wochen des Kurses der Nachbarsprache Polnisch. Dies wäre jedoch nicht ein ausreichendes Argument für die Begründung des Code Switchings ins Russische. Es kann viel mehr angenommen werden, dass der Rückgriff auf das Russische fällt, da die Sprache der slawischen Gruppe angehört und dem Polnischen (sprachtypologisch gesehen) nahe ist.

\begin{tabular}{|l|l|}
\hline Polnischer Arzt: & $\begin{array}{l}\text { Dzień dobry. Jestem lekarzem. Co tutaj mamy? [Guten Tag, Ich bin } \\
\text { Arzt, was haben wir hier?] }\end{array}$ \\
\hline Rettungskraftı: & Nazywam się... Here is my... [eng.] [Ich heiße... Hier ist mein...] \\
\hline Rettungskraft1: & Pacjent alergie insekta (sic!) [Patient hat eine Insektenstichallergie] \\
\hline Rettungskraftı: & Inspiratorisch problem [Atemwegprobleme] \\
\hline Rettungskraft1: & $\begin{array}{l}\text { daj (sic!) tlen, bis [deut.] } 2 \text { mg adrenaliny [wir haben Sauerstoff } \\
\text { gegeben, bis } 2 \text { mg Adrenalin wurde verabreicht] }\end{array}$ \\
\hline Polnischer Arzt: & [stellt eine Frage] \\
\hline Rettungskraft1: & $\begin{array}{l}\text { Piervyj [rus.] (sic!) } 0,5 \text { i. M. and [engl.] patom ... [rus.] [zuerst 0,5 i.M., } \\
\text { dann...] }\end{array}$ \\
\hline Polnischer Arzt: & [stellt eine Frage] \\
\hline Rettungskraft1: & Yes [eng.] i. V. [ja, intravenös] \\
\hline Rettungskraft1: & $\begin{array}{l}\text { oddechowy gut [deut.], patom czetyri litra [rus.] [Atmung in Ordnung, } \\
\text { dann vier Liter] }\end{array}$ \\
\hline
\end{tabular}

TABELLE 2. Übergabe an den Polnischen Arzt vom 4.03.2020 
Auch das Gespräch mit dem polnischen Arzt, wie hier nachgezeichnet, ist von anscheinend nicht-funktionalem Code Switching geprägt. Erneut dominiert das Russische.

Auch wenn sich ein Sprachkompetenzunterschied zwischen den Rettungskräften abgezeichnet hat, da die eine Rettungskraft bilingual war, haben sich die Rettungskräfte gegenseitig bei Kommunikationsproblemen unterstützt. Es wurden auch Selbstkorrekturen erfolgreich vorgenommen.

Wie beobachtet werden konnte, ist der Notarzt an den sprachlich anspruchsvollen Stellen der Übergabe gezielt auf diese eingegangen und hat die notwendige Information erhalten.

\section{a) Simulation Nr. 2}

Die deutschen Rettungskräfte trafen bei einem polnischen Patienten ein, der einen anaphylaktischen Schock hatte. Die Rettungskräfte haben geschafft, sich vorzustellen. Es wurden auch einzelne Lexeme auf Polnisch produziert. Vollständige Sätze auf Polnisch (bis auf einen abgelesenen) blieben aus. In den elliptischen Äußerungen kam es zu Code Switching ins Englische. Parallel erfolgte medizinische Versorgung. Die Rettungskräfte sind beide Erstsprecher des Deutschen und hatten Englisch in der Schule gehabt. Eine der Rettungskräfte hatte auch Russischunterricht.

Da die Ehefrau des Patienten Deutsch konnte, fand die Kommunikation mehrheitlich auf Deutsch statt.

\begin{tabular}{|l|l|}
\hline Rettungskraftı: & Dzien dobry, jestem ratownikiem [Guten Tag, ich bin Notfallsanitäter] \\
\hline Rettungskraft2: & [spricht Deutsch mit dem Patienten] \\
\hline Rettungskraft2: & Prosię, Proszę... [Bitte] \\
\hline Rettungskraftı: & Drogi oddechowe drożne. [Atemwege frei] \\
\hline Rettungskraft2: & Haben Sie [deut.] alergia? [Haben Sie eine Allergie?] \\
\hline Rettungskraftı: & Kiedy pan ostatni raz jadt? [Wann haben Sie gegessen?] \\
\hline Rettungskraftı: & $\begin{array}{l}\text { Jak pojawity si dolegliwości? [Wann sind die Beschwerden } \\
\text { aufgetreten?] }\end{array}$ \\
\hline Rettungskraftı: & hospital [engl.] [Krankenhaus] \\
\hline
\end{tabular}

TABELLE 3. Gespräch mit Patienten vom 11.03.2020

Da die notfallmedizinische Kommunikation mehrheitlich auf Deutsch stattfinden konnte, gab es wenig Bedarf die Nachbarsprache Polnisch zu verwenden.

Es fand ebenso eine Übergabe an einen polnischen Notarzt statt. Wiederum konnte hier Codeswitching ins Russische —vorwiegend bei den Zahlenbeobachtet werden. Weitere Belege für die Übergabe verbaler Besonderheiten liegen nicht vor. Da die Übergabe sehr kurz war und nur die Zahlen notiert wurden, werden die russischen Zahlen hier wiederholt: dva [zwei], pjat' [fünf], szest $^{\prime}$ [sechs]. Hierzu ist es nicht möglich Teilsequenzen zu präsentieren. 


\section{SCHLUSSBEMERKUNGEN}

Die beobachteten und hier beschriebenen Simulationstrainings geben Aufschluss darüber, dass eine Kommunikation nur mit basalen sprachlichen Elementen in der Nachbarsprache Polnisch möglich ist. Die hier abgebildete notfallmedizinische Kommunikation ist jedoch vor allem vom Code Switching geprägt.

Nicht überraschend wäre, wenn der Sprachwechsel mehrheitlich ins Englische stattfinden würde, da viele Begriffe oder Schemata auf dem Englischen basieren, um gemeinsame Kommunikation unter den internationalen Teams zu ermöglichen. Es kommt vereinzelt zu Sprachwechsel ins Englische. Dieser Sprachwechsel ist nicht dominierend und nicht die Kommunikation behindernd.

Im Falle von Code Switching ins Russische wird die Kommunikation nicht erheblich gestört, da die Sequenzen des Sprachwechsels vor allem Zahlen betreffen, die tatsächlich in beiden Sprachen ähnlich ausgesprochen werden können. Dabei ist jedoch zu beachten, dass beobachtete Rettungskräfte zwar vor mehreren Jahren vor dem Simulationstraining einen gesteuerten Russischunterricht beendet haben, jedoch die Sprache und insbesondere ihre Lexeme sich anscheinenden gut im mentalen Lexikon der Teilnehmenden gespeichert haben.

Somit bestätigt sich auch die Annahme, dass die L2 einen Einfluss auf das Erlernen und Produktion von L3 hat, was bei dem Simulationstraining zu beobachten war. Aus kurspraktischen Gründen wäre es sicherlich sinnvoll darüber nachzudenken, inwieweit die Einbindung der Vorkenntnisse des Russischen produktiv für das Erlernen der Nachbarsprache Polnisch wäre. Sicherlich das punktuelle Aufzeigen der Unterschiede zwischen den beiden slawischen Sprachen wäre ebenso produktiv.

Des Weiteren haben die Beobachtungen das Simulationstraining in ein neues noch interessanteres Licht gerückt, das mit möglichen Forschungsdesiderata verbunden ist. In einem gewissen Ausmaß ist es möglich dank den Beobachtungen das sprachliche Handeln auf dem verbalen Niveau zu identifizieren und $\mathrm{zu}$ rekonstruieren, auch wenn nur Audio- oder Videoaufnahmen im Liveübertragungsmodus zur Verfügung standen. Gäbe es eine Möglichkeit dank gespeicherter Videoaufnahmen die ganze kommunikative Situation in den Blick zu nehmen, wären folgende Fragen zu beantworten: 1) Wo begleiten nonverbale Handlungen die Kommunikation, wo substituieren sie sogar verbales Handeln? 2) Kommt es zu offensichtlichen Missverständnissen in der Kommunikation? Wenn ja, werden diese wahrgenommen und welche Maßnahmen werden ergriffen, um die Missverständnisse aufzuklären? 3) Wo scheitert die Kommunikation völlig und woran scheitert sie (undeutliche/falsche Aussprache, grammatische Fehler (Kasus-/Tempus-/Numerusformen), fehlende Lexik in der Nachbarsprache, Nichtbeachten interkulturell relevanter 
pragmatischer Aspekte (Adressierung)? 4) Wie gehen die Probanden mit dem Scheitern der Kommunikation um?

Diese Fragen sind als mögliche Forschungsdesiderata $\mathrm{zu}$ betrachten, die eine Reihe an Untersuchungen benötigen. Die Antworten würden sicherlich auch potenzielle Lösungen für Herausforderungen der komplizierten notfallmedizinischen Kommunikation in einer Nachbarsprache beisteuern können.

\section{LITERATURVERZEICHNIS}

AUER, P. (1988), "A conversation analytic approach to code-switching and transfer", in Codeswitching: Anthropological and Sociological Perspectives, Hell M. (ed.), Berlin, Mouton de Gruyter, 187-213.

BECHMANN, S. (2014), Sprache und Medizin: interdisziplinäre Beiträge zur medizinischen Sprache und Kommunikation, Berlin, Frank \& Timme Verlag für wissenschaftliche Literatur.

BLOM, J.-P. \& GUMPERZ, J. J. (1972), "Social meaning in linguistic structure: codeswitching in Norway", in Directions in Sociolinguistics, Gumperz, J. J. \& Hymes D. (eds.), New York, Holt, Rinehart \& Winston, 407-434.

GeHrAU, V. (2017), Die Beobachtung als Methode in der Kommunikations- und Medienwissenschaft, München, UVK Lucius.

GuMPERZ, J. J. (1982), Discourse Strategies, Cambridge, Cambridge University Press.

HAMMARBERG, B. (2010), "The languages of the multilingual: Some conceptual and terminological issues", IRAL: International Review of Applied Linguistics in Language Teaching, Bd. 48, 91-104.

HRYNIEWICZ, J. \& LISEK, G. (2019), „NNauka języka sąsiada: od kształcenia przedszkolnego do zawodowego. Rozwiązania dydaktyczne i organizacyjne na pomorzu przednim", in Panorama glottodydaktyki polonistycznej. Wyzwania, pytania, kierunki Zioło-Pużuk, K. (ed.), Waszawa, Wydawnictwo UKSW, 149-162.

Hufeisen, B. \& LindeMAnN, B. (1998), Tertiärsprachen: Theorien, Modelle, Methoden, Tübingen, Stauffenburg.

ILLÉS, B. \& KEGYES, E. (2019), „Die Rolle der Simulation im Fachsprachenunterricht“, in Sprachen, Literaturen und Kulturen im Kontakt. Beiträge der 25. Linguistik- und Literaturtage, Miskolc/Ungarn, 2017, Kegyes, E., Kriston, R. \& Schönenberger, M. (Hrsg.), Hamburg, Kovač, 117-126.

KLČOVA, R. (2019), „Die simulierte Hauptverhandlung im Spannungsfeld von linguistischer und juristischer Dimension", in Sprachen, Literaturen und Kulturen im Kontakt. Beiträge der 25. Linguistik- und Literaturtage, Miskolc/Ungarn, 2017, Kegyes, E., Kriston, R. \& Schönenberger, M. (Hrsg.), Hamburg, Kovač, 127-132.

LISEK, G. (2021), „Code-Switching im Kontext von Tertiärsprachenunterricht für deutsch-sprachige Rettungskräfte: Russisch in der Notfallkommunikation im deutsch-polnischen Grenzgebiet", Linguistische Treffen in Wroctaw, 19, 167-185.

LISEK, G. (2020), „Notfallmedizinische Kommunikation im Unterricht Polnisch als Fremdsprache. Analyse von Gesprächsverhalten deutschsprachiger Rettungskräfte", Glottodidactica. An International Journal of Applied Linguistics, XLVII(2), 169-193. 
LISEK, G. (2019), „Fachkommunikation und regionale Vernetzung am Beispiel des Projekts Integrierter grenzüberschreitender Rettungsdienst Pomerania/Brandenburg (InGRiP)“, Greifswalder Beiträge zur Hochschullehre. Perspektiven der Lehre im 21. Jahrhundert, 10, 21-32. Verfügbar unter: https://www.uni-greifswald.de/storages/uni-greifswald/2_Studium/2.1_Studiena ngebot/2.1.4_Qualitaet_in_Studium_und_Lehre/interStudies/Uni_BzH_2019.pdf

MeISEL, J. (2011), First and second language acquisition: parallels and differences, Cambridge, Cambridge University Press.

RIEDL, A. (2012), „Interaktive Lehr-Lern-Methoden: Fallstudie, Rollenspiel, Planspiel, Zukunftswerkstatt und Szenario", in Aktuelle Fragen des Fremdsprachenunterrichts, der interkulturellen Kommunikation und der Fachrichtung Übersetzung an der Hochschule, Korneeva, L. (Hrsg.), Jekaterinburg, UrFU, 104-114.

RIEHL, C. M. (2014), Mehrsprachigkeit: eine Einführung, Darmstadt, WBG.

ROCHE, J. (2013), Fremdsprachenerwerb - Fremdsprachendidaktik, Tübingen, Basel, Francke.

SEARLE, J. R. (1997), Sprechakte: ein sprachphilosophischer Essay, Frankfurt am Main, Suhrkamp.

STEMMLER, J. \& Hecker, U. (2017), Notfallkommando Kommunikation in Notfallsituationen für Gesundheitsberufe, Berlin, Heidelberg, Springer-Verlag. 